\title{
EFFECTS OF DUAL RENIN-ANGIOTENSIN SYSTEM BLOCKADE ON PROTEINURIA IN A HYPERTENSIVE BLACK AFRICAN HIV- INFECTED PATIENT
}

\author{
Claudio Ucciferri ${ }^{1}, M D$ \\ Katia Falasca ${ }^{1}, M D$ \\ Paola Mancino ${ }^{1}, M D$ \\ Roberto Tommasi ${ }^{2}, M D$ \\ Alfonso Tatasciore ${ }^{2}, M D$ \\ Jacopo Vecchiet ${ }^{1}, M D$ \\ ${ }^{1}$ Infectious Disease Clinic - Department of Medicine and Science of Aging, G d'Annunzio University, Chieti, Italy \\ ${ }_{2}^{2}$ Institute of Cardiology, Center of Excellence on Aging, G d'Annunzio University
}

Kidney diseases manifesting as proteinuria or elevated creatinine are increasingly prevalent complications of HIV infection. We report the effects of dual renin-angiotensin system blockade on proteinuria in a hypertensive black African HIV-infected patient.

Kidney diseases manifesting as proteinuria or elevated creatinine are increasingly prevalent complications of HIV infection. Today HIV-associated nephropathy (HIVAN) is a main cause of end-stage renal disease in HIV-infected African-Americans, and it is likely to be a result of a recently identified genetic predisposition based on polymorphisms of the myosin heavy-chain 9 (MYH9) gene. ${ }^{1}$ However, a variety of other histopathological renal diseases affect HIV-infected subjects of all ethnic groups. ${ }^{1}$ HIV-associated renal disease with overt proteinuria has been associated with poorer outcomes and increased mortality. ${ }^{2}$ Moreover, an increased rate of urinary albumin excretion, even in the micro-albuminuric range, is an indicator of glomerular damage and has been found to be associated with an increased risk of cardiovascular disease (CVD) and mortality in the general population. ${ }^{2}$

The pathophysiological mechanisms underlying urinary albumin excretion and the increased risk of CVD are not fully understood. Both HIV-related and non-HIVrelated factors may play a role. There are few studies of albuminuria in HIV-infected patients, and most of these have been undertaken in selected small cohorts limited to the pre-combination antiretroviral therapy (CART) era.

The renin-angiotensin system (RAS) is a major regulator of blood pressure and vascular response to injury. Continual activation of the RAS is associated with hypertension and end-organ damage, including renal disease and CVD. Inhibitors of the RAS have become a cornerstone for the treatment of hypertension. Use of these agents in patients with underlying renal disease has revealed that RAS inhibition exerts an anti-proteinuric effect independent of blood pressure reduction. ${ }^{3}$ Because RAS intervention can be targeted at various points, it has been postulated that combining more than one of these intervention points could lead to more effective inhibition of the RAS and a more robust decrement in protein excretion. Several studies support the use of combination therapy with angiotensinconverting enzyme inhibitors (ACE-Is) and angiotensin receptor blockers (ARBs) in the general population, ${ }^{4}$ but there are no data on dual RAS blockade effects in HIVpositive patients with proteinuria.

\section{CASE REPORT}

A 45-year-old HIV-infected black African man (CDC A2) came to the Infectious Disease Clinic at SS Annunziata Hospital, Chieti, Italy, for follow-up. He had been treated with tenofovir + emtricitabine + efavirenz for one year at another centre in Italy. His personal history included multiple sexual partners, use of illegal injected drugs, tobacco smoking and chronic HCV infection. On admission the CD4+ level was 603 cells/ $\mu$ l, the CD4/ CD8 ratio 0.44 , the HIV-RNA level $<40$ copies $/ \mathrm{ml}$, and the HCV-RNA level $>5 \times 10^{5}$ copies $/ \mathrm{ml}$. Levels of the transaminases and glucose and lipid parameters were within normal limits, the glomerular filtration rate (GFR) assessed by the MDRD logarithmic model (MDRD-GFR) was $114 \mathrm{ml} / \mathrm{min} / 173 \mathrm{~m}^{2}$, and the urinary albumin level was $1.5 \mathrm{~g} / \mathrm{l}$. The blood pressure (BP), measured with a mercury sphygmomanometer with an appropriately sized cuff after the patient had rested in a seated position, was elevated at $150 / 100 \mathrm{mmHg}$. Treatment with 10 $\mathrm{mg}$ lercanidipine daily was started, strictly monitoring possible side-effects and interactions with cART. After 1 month of antihypertensive therapy the BP had fallen to 
$120 / 90 \mathrm{mmHg}$ without adverse effects, while the urinary albumin level and MDRD-GFR were $1.21 \mathrm{~g} / \mathrm{l}$ and $106 \mathrm{ml} /$ $\mathrm{min} / 173 \mathrm{~m}^{2}$, respectively. A change in cART, withdrawing tenofovir, was proposed, but the patient refused it. As a consequence, in spite of the improved mean BP (130/85 $\mathrm{mmHg}$ ), the albuminuria progressively worsened over the following months (to $2.52 \mathrm{~g} / \mathrm{l}$, with the MDRD-GFR rising to $117 \mathrm{ml} / \mathrm{min} / 173 \mathrm{~m}^{2}$ ). The patient refused renal biopsy or cART changes. Administration of $300 \mathrm{mg}$ irbesartan daily was then started in order to improve the renal dysfunction; after a month, while the BP and MDRD-GFR remained stable, the urinary albumin level had decreased only to $150 \mathrm{mg} / \mathrm{dl}$. We therefore added lisinopril $20 \mathrm{mg} / \mathrm{d}$ to achieve dual blockade of the RAS. After 5 months the urinary albumin level had fallen to $0.285 \mathrm{~g} / \mathrm{l}$ without any change in BP, MDRD-GFR or viroimmunological parameters (Fig. 1).

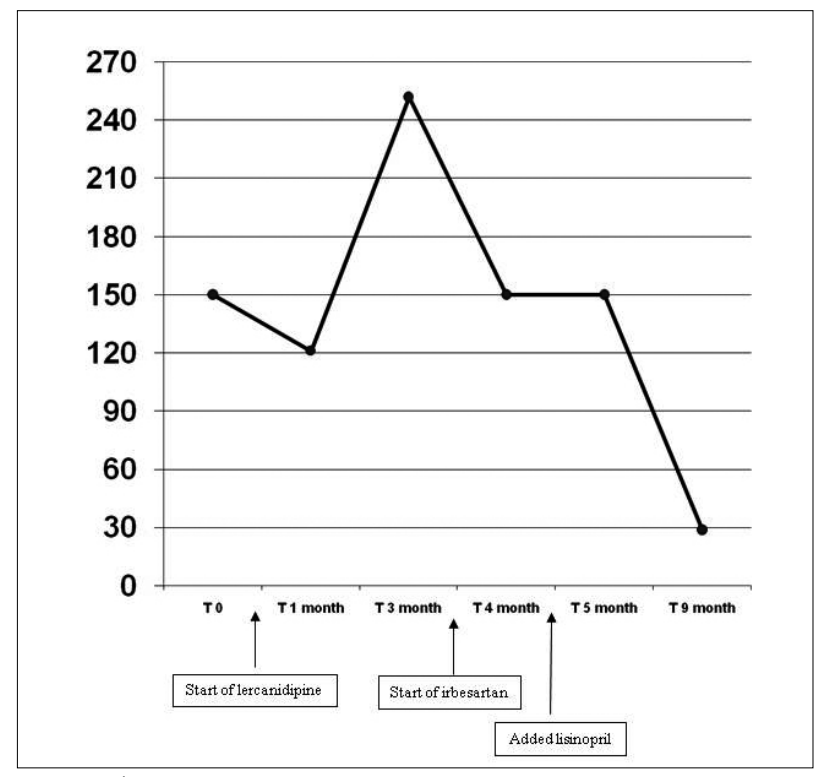

Fig. 1. Urinary albumin levels over the treatment period.

\section{DISCUSSION}

HIV infection is a strong risk factor for renal disease because of the presence of proteinuria, independent of other risk factors. Previously reported risk factors for HIV-related proteinuria include both traditional HIV-specific markers, such as CD4 lymphocyte count and HIV RNA levels, and several traditional renal and cardiovascular risk factors, such as higher systolic BP and insulin resistance. African-Americans, older people and those with a lower GFR and hepatitis $C$ co-infection are also at increased risk. ${ }^{1}$ The potential nephrotoxicity induced by CART may considerably increase the risk of renal disease. Although previous studies of the longitudinal effects of cART on proteinuria are limited, certain frequently used antiretroviral medications such as tenofovir have been associated with acute renal failure. Moreover, there is increasing evidence that HIV-infected patients have hypertension requiring pharmacological treatment, which also represents an important risk factor for kidney disease.

The choice of antihypertensive therapy for HIV-infected cART-treated patients must be made carefully. The classes of antihypertensive drugs that block the RAS, such as ACE-Is and ARBs, may be ideal. Several studies have reported beneficial effects of dual RAS blockade on kidney disease progression and proteinuria reduction in the general population. A more recent trial (IMPROVE) ${ }^{5}$ has shown the effectiveness of fixed-dose combination ARB/ACE-I therapy in reducing albuminuria in non-HIVinfected hypertensive subjects. It is therefore advisable to use combination ARB/ACE-I therapy in more complex cases to obtain a greater nephroprotective effect. Data on ACE-I and ARB use in HIV-infected patients are anecdotal, ${ }^{6}$ and there have been no well-designed studies on this subject to date.

Our patient's race was an additional problem in selecting effective antihypertensive therapy, because antihypertensive regimens that inhibit the RAS may be ineffective in black patients. In the African-American non-infected population, responsiveness to monotherapy with ACE-Is, ARBs and beta-blockers may be poorer than responsiveness to diuretics and calcium channel blockers (CCBs). ${ }^{7}$ In our patient dual RAS blockade therapy did not have substantial effects on BP control, while the main antihypertensive effect was achieved with the use of the CCB lercanidipine, in the absence of important interactions with CART. Lercanidipine did not improve proteinuria in our patient. This may indicate that HIV-related kidney disease is not only correlated with hypertension levels. The use of combined therapy with $20 \mathrm{mg}$ lisinopril plus $300 \mathrm{mg}$ irbesartan, obtaining a dual RAS blockade, produced a marked improvement in kidney function, with a $-88 \%$ decrease from the baseline urinary albumin level.

\section{CONCLUSION}

This case shows that dual blockade of the RAS with combined ACE-I and ARB therapy reduced albuminuria in an HIV-infected black African patient. Furthermore, it indicates that the lowering of protein excretion brought about by dual RAS blockade is independent of BP reduction. Available data suggest that the beneficial effect achieved with combination therapy derives from more prolonged and complete inhibition of the RAS rather than from a physiological interaction between the ACE-I and the ARB. ${ }^{4}$ This more extensive RAS inhibition may provide incremental end-organ protection through its effects on chronic vascular responses to injury.

There are no conflicts of interest. No financial support was given for the study.

\section{REFERENCES}

1. Szczech LA, Gupta SK, Habash R, et al. The clinical epidemiology and course of the spectrum of renal diseases associated with HIV infection. Kidney Int 2004;66:1145-1152.

2. Hillege HL, Fidler $V$, Diercks GF, et al. Urinary albumin excretion predicts cardiovascular and noncardiovascular mortality in general population. Circulation 2002;106:1777-1782.

3. Berl T. Renal protection by inhibition of the renin-angiotensin-aldosterone system. J Renin Angiotensin Aldosterone Syst 2009;10:1-8.

4. Linas SL. Are two better than one? Angiotensin-converting enzyme inhibitors plus angiotensin receptor blockers for reducing blood pressure and proteinuria in kidney disease. Clinical Journal of the American Society of Nephrology 2008;3:S17-S23.

5. Bakris GL, Ruilope L, Locatelli F, et al. Treatment of microalbuminuria in hypertensive subjects with elevated cardiovascular risk: results of the IMPROVE trial. Kidney Int 2007;72(7):879-885.

6. Ucciferri C, Mancino P, Vecchiet J, Falasca K. Beneficial effects of telmisartan in HIV+ diabetic insulin-dependent patient. Int J Immunopathol Pharmacol 2009;22:853-858.

7. Ferdinand KC, Armani AM. The management of hypertension in African Americans. Critical Pathways in Cardiology 2007;6:67-71. 